\title{
The Simultaneous Elevation of Oxidative Stress Markers and Wilms' Tumor 1 Gene during the Progression of Myelodysplastic Syndrome
}

\author{
Naomi Shimizu ${ }^{1}$, Hidekazu Hasunuma ${ }^{1}$, Yasuhiro Watanabe ${ }^{2}$, Yasuo Matsuzawa ${ }^{3}$, \\ Youichi Iwashita ${ }^{1}$, Ichiro Tatsuno ${ }^{2}$ and Hiromitsu Yokota ${ }^{4}$
}

\begin{abstract}
Oxidative stress is closely related to iron overload in myelodysplastic syndrome (MDS) and induces DNA damage. We evaluated the oxidative stress markers derivatives of reactive oxidative metabolites (dROM) and 8-hydroxy-2'-deoxyguanosine (8-OHdG) during azacitidine treatment in an MDS patient. Simultaneous with an increase in the expression of Wilms' Tumor 1 (WT1) gene in the peripheral blood, the serum dROM level was elevated, and this increase was observed earlier than the increases in ferritin and 8-OHdG. Throughout the clinical course, dROM and 8-OHdG correlated significantly with WT1 and with ferritin, suggesting that changes in the oxidative stress marker levels reflect not only iron overload but also disease progression of MDS.
\end{abstract}

Key words: myelodysplastic syndrome, azacitidine, oxidative stress, ferritin, Wilms' Tumor 1 (WT1)

(Intern Med 55: 3661-3664, 2016)

(DOI: 10.2169/internalmedicine.55.7156)

\section{Introduction}

DNA damage caused by oxidative stress plays an important role in the initiation and carcinogenic process of mutation. A recent study has suggested that oxidative stress leads to increased mutation frequency in a murine model of myelodysplastic syndrome (MDS) (1), and that additional mutations lead to the progression of MDS. Patients with MDS often require blood transfusions and consequently develop iron overload, and the excess iron causes oxidative stress. Therefore, antioxidant therapy and iron chelation are thought to be suitable for the management of MDS patients (2-4). Furthermore, a correlation has been reported between the serum ferritin level and serum reactive oxygen species (ROS) levels in MDS patients (2-5). MDS is a heterogeneous group of clonal hematopoietic stem cell disorderd that transforms to acute myeloid leukemia (6). DNA hypermethylation is a major pathogenesis of MDS (7). Azacitidine was the first
DNA-hypomethylating agent that demonstrated superiority over conventional therapy in the treatment of MDS in patients unable to undergo allogeneic stem cell transplantation (8). A relationship between oxidative stress and hypermethylation has also been reported; oxidative stress is likely involved in the MDS disease progression (9). In the present study, we evaluated the changes in the oxidative stress markers derivatives of reactive oxidative metabolites (dROM) in serum and 8-hydroxy-2'-deoxyguanosine (8-OHdG) in urine in an MDS patient treated with azacitidine. The Ethics Committee of Toho University approved this study, and we obtained informed consent from the patient.

\section{Case Report}

A 69-year-old Japanese man with exertional chest pain was transported to our hospital by ambulance in April 2014. He had a history of unstable angina and had undergone percutaneous coronary intervention at the left anterior descend-

\footnotetext{
${ }^{1}$ Department of Blood Transfusion, Toho University Medical Center Sakura Hospital, Japan, ${ }^{2}$ Center for Diabetes, Metabolism and Endocrinology, Toho University Medical Center Sakura Hospital, Japan, ${ }^{3}$ Department of Internal Medicine, Toho University Sakura Medical Center, Japan and ${ }^{4}$ Clinical Laboratory Program, Education Development Center, Faculty of Science, Toho University, Japan Received for publication January 19, 2016; Accepted for publication April 24, 2016 Correspondence to Dr. Naomi Shimizu, naomis-cib@umin.ac.jp
} 


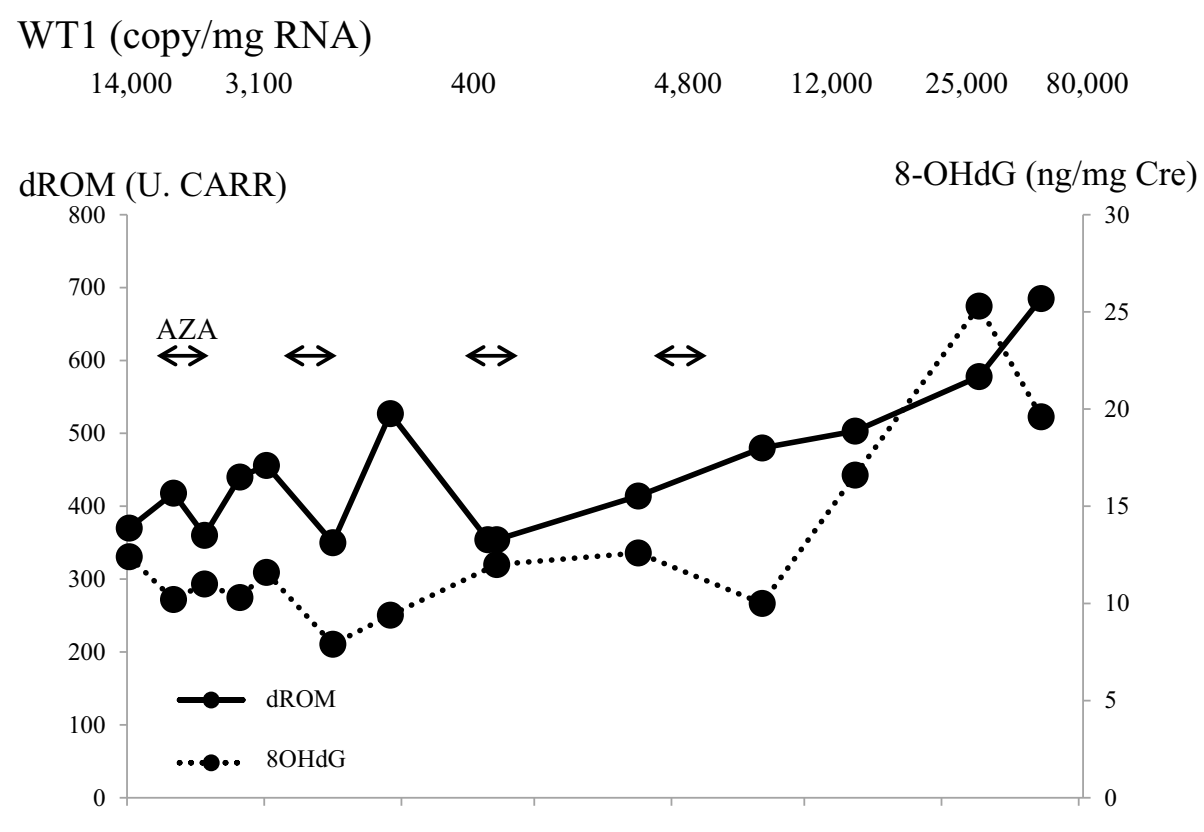

FER $(\mathrm{ng} / \mathrm{mL})$

LDH (IU/L)

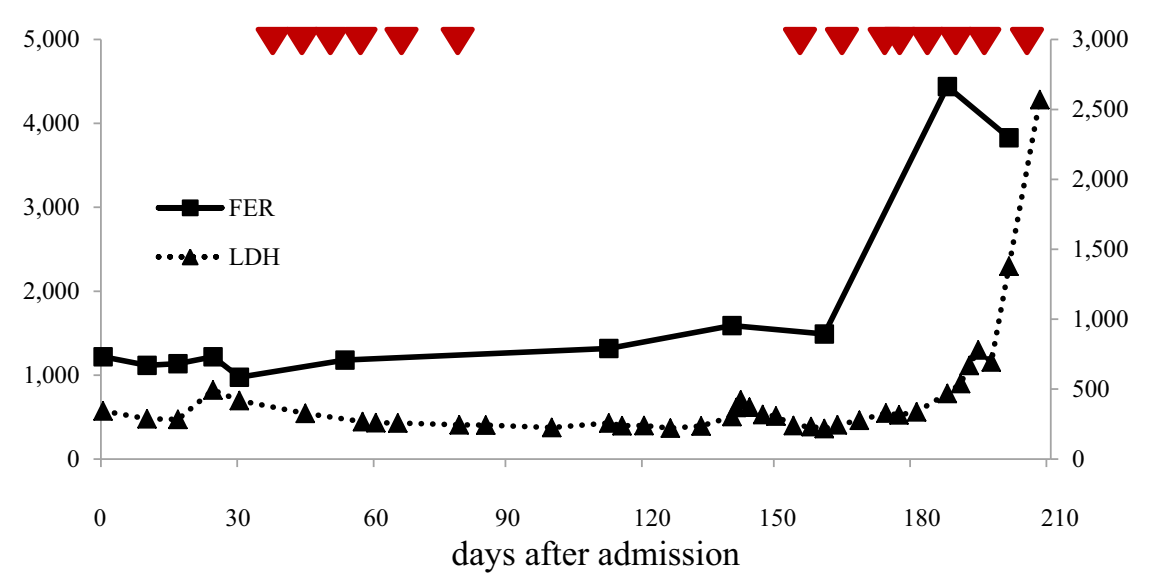

Figure 1. The clinical course of the patient. AZA: azacitidine, WT1: Wilms' Tumor 1, dROM: derivatives of reactive oxygen metabolite, 8-OHdG: 8-hydroxy- 2'-deoxyguanosine, FER: ferritin, $(\nabla)$ : red blood cell transfusion 2 units. Normal ranges: serum dROM; <300 U. CARR, urinary 8-OHdG; $<15.7 \mathrm{ng} / \mathrm{mg}$ creatinine ferritin; 18.6-261 ng/dL, lactate dehydrogenase (LDH); 120-240 IU/L.

ing artery in November 2013. On admission, the echocardiogram and laboratory tests showed no findings of ischemic heart disease. However, his white blood cell (WBC) count was $1,970 / \mu \mathrm{L}$ without blast cells; his hemoglobin $(\mathrm{Hb})$ level was $5.0 \mathrm{~g} / \mathrm{dL}$; and his platelet count was $16.6 \times 10^{4} / \mu \mathrm{L}$. A bone marrow examination revealed myelodysplastic syndrome classified as refractory anemia with excess blasts-1 (RAEB-1). He also had a markedly complicated karyotype including trisomy 8. Initially, metenolone enanthate and menatetrenone were administered, and red blood cell transfusion was performed to treat his anemia. He was admitted to our hospital at the end of July 2014, and azacitidine therapy was started on Day 3 after admission. After the fourth course of azacitidine therapy, he developed acute myocardial infarction on Day 140. Azacitidine therapy was discontinued, and therapy with the iron chelator deferasirox $500 \mathrm{mg}$ / day was started on Day 192. A total of 28 units of blood had been transfused before starting administration of the iron chelator.

We evaluated the levels of $\mathrm{dROM}$ in serum by colorimetry (10) and 8-OHdG in spot urine by ELISA method (11), ferritin in serum, and Wilms' Tumor 1 (WT1) gene in the peripheral blood throughout the course of treatment (Fig. 1). The level of WT1 was 14,000 copies/ $\mu \mathrm{g}$ RNA before the first course of azacitidine treatment, which was reduced markedly to 400 copies/ $\mu$ g RNA after the second course. However, the level of WT1 increased sharply around the fourth course of azacitidine therapy, and pancytopenia with blasts in the peripheral blood became gradually apparent. The respective serum $\mathrm{dROM}$ and urinary $8-\mathrm{OHdG}$ levels were 418 U.CARR and $10.2 \mathrm{ng} / \mathrm{mg}$ creatinine before the first course of azacitidine treatment and 360 U.CARR and $11 \mathrm{ng} / \mathrm{mg}$ creatinine after the first course. The serum dROM level decreased transiently with each course of azacitidine 

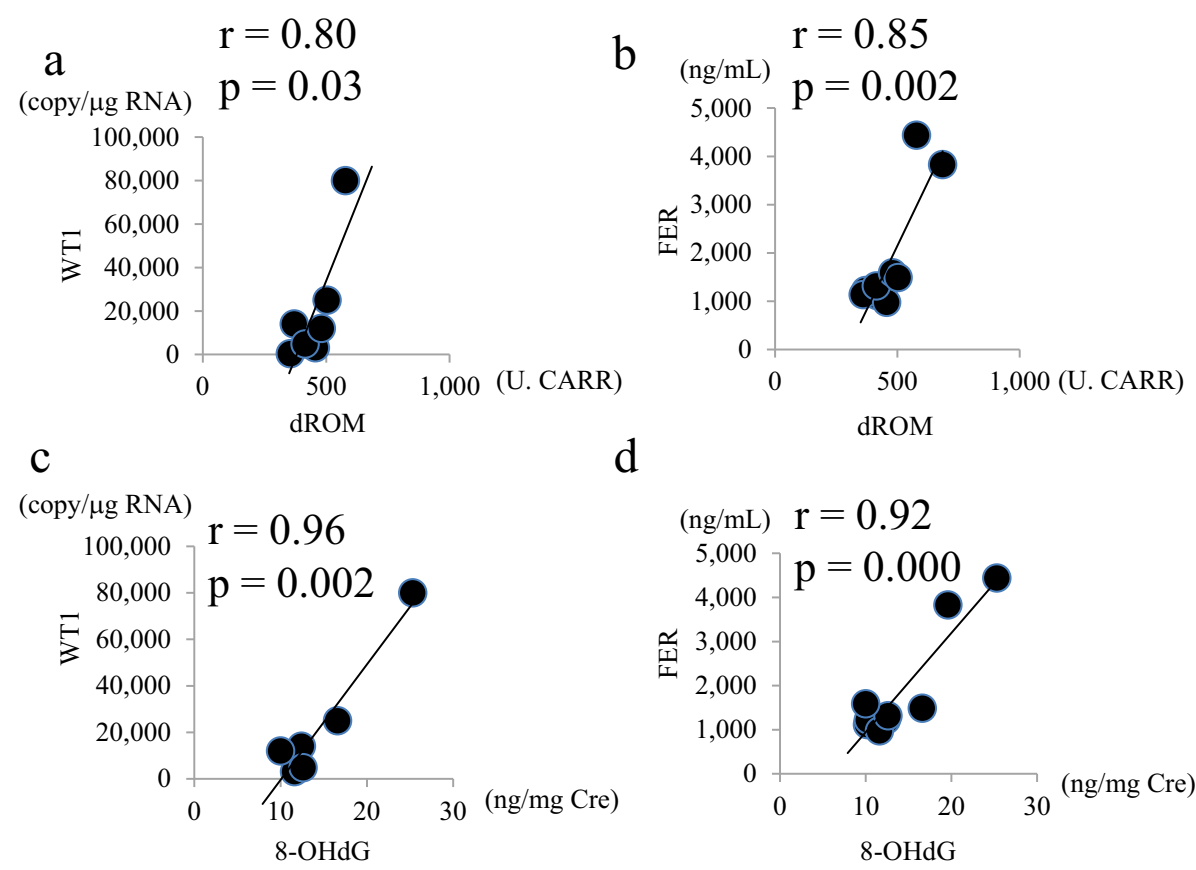

d

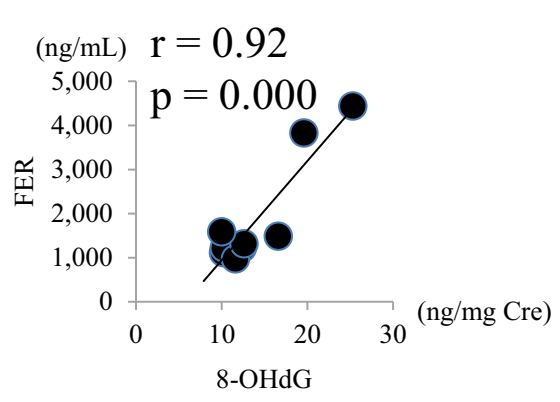

e

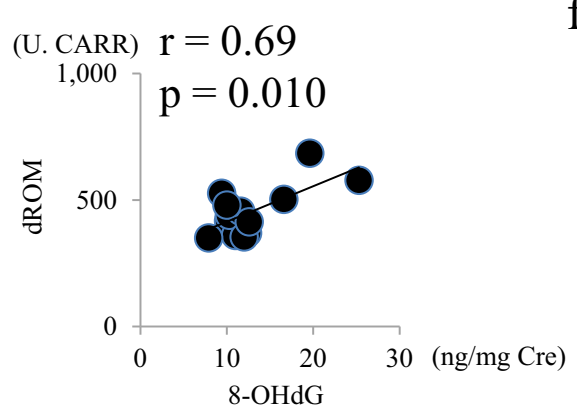

f

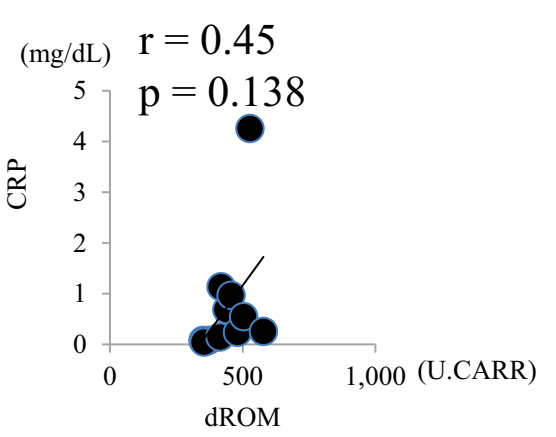

g

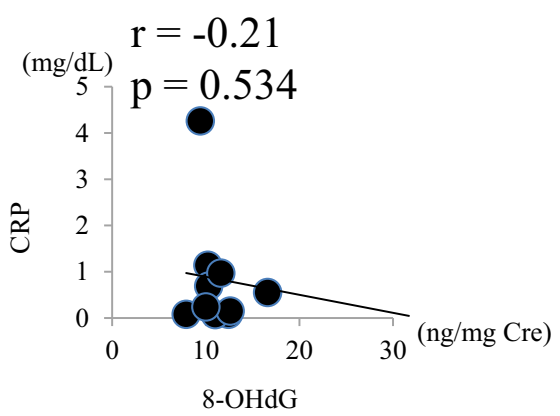

Figure 2. The relationship among the derivatives of reactive oxygen metabolite (dROM), 8-hydroxy-2'-deoxyguanosine (8-OHdG), ferritin (FER), and Wilms' Tumor 1 (WT1). The correlation between (a) dROM and WT1, (b) dROM and FER, (c) 8-OHdG and WT1, (d) 8-OHdG and FER, (e) 8-OHdG and dROM, (f) dROM and C reactive protein (CRP), and (f) 8-OHdG and CRP.

therapy until the third course. From the fourth course, accompanying an increase in WT1, dROM increased simultaneously while 8-OHdG and ferritin began to increase later.

Around Day 190 after the first admission, the WBC count of the peripheral blood showed $30 \%$ blasts, and the serum dROM, urinary 8-OHdG, and serum ferritin levels were elevated markedly to peak levels of 685 U.CARR, $25.3 \mathrm{ng} / \mathrm{mg}$ creatinine, and 4,440.3 ng/mL, respectively. He was admitted to the hospital on Day 192 due to a deterioration of general condition. He received circulatory support and blood transfusion and was treated with deferoxamine mesilate at $500 \mathrm{mg} /$ day. His disease progressed, and he eventually died of multi-organ failure on Day 210.

We analyzed the associations among dROM, 8-OHdG, ferritin, and WT1 levels during his clinical course by Pearson's test. The serum dROM levels correlated significantly with WT1 ( $\mathrm{r}=0.80, \mathrm{p}=0.03)$ and ferritin $(\mathrm{r}=0.85, \mathrm{p}=0.002)$ (Fig. 2a and b). In addition, the urinary $8-\mathrm{OHdG}$ levels also correlated significantly with WT1 $(r=0.96, p=0.002)$ and ferritin $(r=0.92, p=0.000)$ (Fig. $2 c$ and $d)$. The serum $d R O M$ 
levels also correlated significantly with $8-\mathrm{OHdG}(\mathrm{r}=0.69, \mathrm{p}=$ 0.01 ) (Fig. 2e). On analyzing the correlation among $\mathrm{C}$ reactive protein (CRP), dROM, and $8-\mathrm{OHdG}$, CRP did not correlate with $\mathrm{dROM}(\mathrm{r}=0.45, \mathrm{p}=0.138)$ or $8-\mathrm{OHdG}(\mathrm{r}=-0.21$, $\mathrm{p}$ $=0.534)$ (Fig. $2 \mathrm{f}$ and $\mathrm{g}$ ).

\section{Discussion}

We encountered a patient with MDS classified as RAEB1 , in whom we measured oxidative stress markers, ferritin, and WT1 over the course of disease. To our knowledge, this is the first report of azacitidine therapy in an MDS patient with serial measurements of oxidative stress, ferritin, and WT1 values during therapy. A marked decline in the WT1 expression in peripheral blood showed that three courses of azacitidine therapy were effective in suppressing disease progression. Furthermore, the serum dROM levels decreased transiently with each course. Oxidative stress and ferritin are considered important biological factors in the pathogenesis of MDS (2-4). However, as shown in Fig. 1, the WT1 expression started to increase before the administration of the fourth course of azacitidine therapy, and the serum dROM levels began to rise earlier than ferritin during disease progression.

Fig. 1 shows that the response of dROM appeared to be more sensitive than that of 8 -OHDG. However, both dROM and $8-\mathrm{OHdG}$ showed strong correlation with WT1 (Fig. 2a and c). We also evaluated the correlation among dROM, 8-OHdG, and the total number of blasts in the peripheral blood. While the dROM levels and the blast count correlated significantly, the $8-\mathrm{OHdG}$ levels showed no correlation with the blast count (data not shown). Ferritin also correlated closely with dROM and 8-OHdG (Fig. 2b and d). On investigating the source of the increased ROS expression during disease progression in this patient by examining the ROS in each fraction of WBCs, the results suggested that the oxidative stress markers were produced by tumor-derived cells (data not shown). Furthermore, to clarify the relationship between the elevation of the oxidative stress markers with the deterioration of the general condition, we also analyzed the correlation among CRP, dROM, and 8-OHdG. CRP did not correlate with either of the oxidative stress markers (Fig. 2f and g).

Azacitidine is a methylation inhibitor. However, in the present case, we observed a transient decreasing trend in the dROM levels after each course of azacitidine treatment before the progression of MDS, and the transition of dROM was not related to the iron overload caused by blood transfusion. Niu et al. recently reported a relationship between oxidative stress and DNA methylation, suggesting that oxidative stress modulates the enzyme activity of DNA methylation and the deacetylation of histones (9). It remains unclear whether the increase in oxidative stress is a cause or a result of disease progression. The increase in the oxidative stress markers may have not only caused the iron overload but also resulted in the disease progression of MDS. Further study will thus be required to clarify this point.

We herein reported an MDS patient who showed simultaneous increases in oxidative stress markers and WT1 during disease progression while being treated with azacitidine. We speculate that the increase in the oxidative stress markers is a cause of not only iron overload but also the disease progression of MDS.

The authors state that they have no Conflict of Interest (COI).

\section{References}

1. Chung YJ, Robert C, Gough SM, Rassool FV, Aplan PD. Oxidative stress leads to increased mutation frequency in a murine model of myelodysplastic syndrome. Leuk Res 38: 95-102, 2014.

2. Ghoti H, Amer J, Winder A, Rachmilewitz E, Fibach E. Oxidative stress in red blood cells, platelets and polymorphonuclear leukocytes from patients with myelodysplastic syndrome. Eur J Haematol 79: 463-467, 2007.

3. Saigo K, Takenokuchi M, Hiramatsu Y, et al. Oxidative stress levels in myelodysplastic syndrome patients: their relationship to serum ferritin and haemoglobin values. J Int Med Res 39: 19411945, 2011.

4. Kikuchi S, Kobune M, Iyama S, et al. Improvement of ironmediated oxidative DNA damage in patients with transfusiondependent myelodysplastic syndrome by treatment with deferasirox. Free Radic Biol Med 53: 643-648, 2012.

5. Gonçalves AC, Cortesão E, Oliveiros B, et al. Oxidative stress and mitochondrial dysfunction play a role in myelodysplastic syndrome development, diagnosis, and prognosis: a pilot study. Free Radic Res 49: 1081-1094, 2015.

6. Tanaka H, Shimizu N, Tougasaki E, et al. Successful treatment by azacitidine therapy of intestinal Behçet's disease associated with myelodysplastic syndrome. Int J Hematol 97: 520-524, 2013.

7. Goncalves AC, Cortesao E, Oliveiros B, et al. Oxidative stress levels are correlated with P15 and P16 gene promoter methylation in myelodysplastic syndrome patients. Clin Exp Med 49: 1081-1094, 2015.

8. Fenaux P, Mufti GJ, Hellstrom-Lindberg E, et al. Efficacy of azacitidine compared with that of conventional care regimens in the treatment of higher-risk myelodysplastic syndromes: a randomised, open-label, phase III study. Lancet Oncol 10: 223-232, 2009.

9. Niu Y, DesMarais TL, Tong Z, Yao Y, Costa M. Oxidative stress alters global histone modification and DNA methylation. Free Radic Biol Med 82: 22-28, 2015.

10. Vassalle C, Boni C, Di Cecco P, Ndreu R, Zucchelli GC. Automation and validation of a fast method for the assessment of in vivo oxidative stress levels. Clin Chem Lab Med 44: 1372-1375, 2006.

11. Saito S, Yamauchi H, Hasui Y, et al. Quantitative determination of urinary 8-hydroxydeoxyguanosine (8-OH-dg) by using ELISA. Res Commun Mol Pathol Pharmacol 107: 39-44, 2000.

The Internal Medicine is an Open Access article distributed under the Creative Commons Attribution-NonCommercial-NoDerivatives 4.0 International License. To view the details of this license, please visit (https://creativecommons.org/licenses/ by-nc-nd/4.0/).

(C) 2016 The Japanese Society of Internal Medicine

http://www.naika.or.jp/imonline/index.html 